\title{
ON GROWTH IN MINIMAL TOTALLY ACYCLIC COMPLEXES
}

\author{
PETTER ANDREAS BERGH AND DAVID A. JORGENSEN
}

\begin{abstract}
Given a commutative Noetherian local ring, we provide a criterion under which a minimal totally acyclic complex of free modules has symmetric growth. As a special case, we show that, whenever an image in the complex has finite complete intersection dimension, then the complex has symmetric polynomial growth.
\end{abstract}

1. Introduction. Given a commutative Noetherian local ring $A$, when does a minimal totally acyclic complex of free modules have symmetric growth? In other words, given such a complex, does the left growth of the ranks of its free modules equal the right growth? For $M$, the image of a differential in such a complex, the left growth may be measured by the complexity of $M$, whereas the right growth may be measured by the complexity of its dual $M^{*}=\operatorname{Hom}_{A}(M, A)$. In this paper, we study symmetric growth in the sense that both these invariants are finite and equal to one another:

$$
\mathrm{cx}_{A} M=\operatorname{cx}_{A} M^{*} .
$$

Avramov and Buchweitz show in [4] that this is always the case for minimal totally acyclic complexes of free modules over local complete intersections. However, Jorgensen and Şega showed in [20] that it does not hold for a local ring in general, even when the ring is Gorenstein. In fact, they constructed such a ring and a minimal totally acyclic complex whose left growth is exponential and right growth is constant. (The characteristics of growth in the dual complex are thus reversed.)

In this paper, we give a criterion under which symmetric growth of minimal totally acyclic complexes holds. This criterion is given in

2010 AMS Mathematics subject classification. Primary 13D07, 13D25, 18 E30.

Keywords and phrases. Totally acyclic complexes, symmetric growth, finitely generated cohomology.

The first author was supported by NFR Storforsk Grant no. 167130. The second author was supported by the Algebra Group at the Institutt for Matematiske Fag, NTNU.

Received by the editors on July 3, 2012, and in revised form on August 21, 2012. DOI:10.1216/JCA-2014-6-1-17 Copyright (C)2014 Rocky Mountain Mathematics Consortium 
terms of the cohomology of the image of a given differential in the complex. Namely, we show in Section 3 that if the cohomology is finitely generated with respect to a ring acting centrally on the derived category, and the ring action commutes with dualization, then the complex has symmetric polynomial growth. In Section 4 we show that this criterion is satisfied in the case of minimal totally acyclic complexes of free modules over complete intersections, and thus we recover the result referred to above. In fact, it is enough to know that an image in the complex has finite complete intersection dimension in order to conclude symmetric polynomial growth. In Section 2 we discuss the necessary preliminaries.

2. Preliminaries. Fix a local (meaning commutative Noetherian local) ring $(A, \mathfrak{m}, k)$, and consider a complex

$$
C: \cdots \longrightarrow C_{2} \stackrel{d_{2}^{C}}{\longrightarrow} C_{1} \stackrel{d_{1}^{C}}{\longrightarrow} C_{0} \stackrel{d_{0}^{C}}{\longrightarrow} C_{-1} \stackrel{d_{-1}^{C}}{\longrightarrow} C_{-2} \longrightarrow \cdots
$$

of finitely generated free $A$-modules. The complex $C$ is called totally acyclic if it is exact, and the dual complex $C^{*}=\operatorname{Hom}_{A}(C, A)$ is also exact. The complex $C$ is called minimal (cf., [7]) if $\operatorname{Im} d_{n}^{C} \subseteq \mathfrak{m} C_{n-1}$ for all $n \neq 0$. Thus, we say $C$ is a minimal totally acyclic complex if it has both of these properties.

The goal of this paper is to study the rate of growth of the ranks of the free modules $C_{n}$ for $n \geq 0$ versus that of the free modules $C_{n}$ for $n \leq-1$, for a given minimal totally acyclic complex $C$. Below we reformulate this into the study of certain modules.

Minimal resolutions. For a complex $C$, we let $M_{C}$ denote the module $\operatorname{Im} d_{0}^{C}$. If $C$ is a minimal totally acyclic complex, then $C_{\geq 0}$ is a minimal free resolution of $M_{C}$, where $C_{\geq 0}$ is the complex with $\left(C_{\geq 0}\right)_{n}=C_{n}$ for $n \geq 0$ and $\left(C_{\geq 0}\right)_{n}=0$ for $n \leq-1$. With the indexing convention $\left(\bar{C}^{*}\right)_{n}=\left(C_{-n-1}\right)^{*}$ and $d_{n}^{C^{*}}=\left(d_{-n}^{C}\right)^{*}$ for all $n$, it follows directly from the properties of a minimal totally acyclic complex that $\left(C^{*}\right)_{\geq 0}$ is a minimal free resolution of $\left(M_{C}\right)^{*}$. Therefore, for all $i \in \mathbf{Z}$, we have $\Omega_{A}^{i}(M)^{*}=\Omega_{A}^{-i}\left(M^{*}\right)$, where $\Omega_{A}^{i}(M)$ denotes $\operatorname{Im} d_{i}^{C}$, and similarly for $\Omega_{A}^{i}\left(M^{*}\right)$.

Recall that the Betti numbers $\beta_{n}(M)$ of an $A$-module $M$ are the ranks of the free modules in a minimal free resolution of $M$. Thus, the 
Betti numbers $\beta_{n}\left(M_{C}\right)$ of $M_{C}$ and $\beta_{n}\left(\left(M_{C}\right)^{*}\right)$ of $\left(M_{C}\right)^{*}$ are given by the ranks of the free modules in $C_{\geq 0}$ and $\left(C^{*}\right)_{\geq 0}$, respectively, for all $n \geq 0$, and so growth in the ranks of the free modules in $C$ for $n \geq 0$ and $n \leq-1$ is the same as the growth of the Betti sequences $\left\{\beta_{n}\left(M_{C}\right)\right\}$ and $\left\{\beta_{n}\left(\left(M_{C}\right)^{*}\right)\right\}$.

There is a name for modules $M$ of the form $M \cong M_{C}$ for $C$ a minimal totally acyclic complex (see [2]).

Definition 2.1. A finitely generated $A$-module $M$ is of Gorenstein dimension zero, or G-dimension zero for short, (or totally reflexive) if $M \cong M_{C}$ for some minimal totally acyclic complex $C$.

Let $M$ be a finitely generated $A$-module, and recall that the complexity of $M[\mathbf{3}]$, denoted $\operatorname{cx}_{A} M$, is defined as

$$
\begin{aligned}
\operatorname{cx}_{A} M \stackrel{\text { def }}{=} & \inf \{t \in \mathbf{N} \cup\{0\} \mid \\
& \text { there exists } \left.a \in \mathbf{R} \text { such that } \beta_{n}(M) \leq a n^{t-1} \text { for } n \gg 0\right\} .
\end{aligned}
$$

In other words, the complexity of $M$ measures the polynomial rate of growth of the Betti numbers of $M$.

The aim of this paper is to give sufficient conditions for a minimal totally acyclic complex $C$ to have symmetric polynomial growth. Given the discussion above, we formulate this task as the following.

Goal. Given an $A$-module $M$ of Gorenstein dimension zero, find sufficient conditions on $M$ and $M^{*}$ such that

$$
\operatorname{cx}_{A} M=\operatorname{cx}_{A} M^{*}<\infty \text {. }
$$

The main apparatus in accomplishing this task is the existence of a ring acting on cohomology. We make the necessary statements in the generality of the bounded derived category $D^{b}(A)$ of $A$; one is welcome to think only of the case of modules in the discussion below. Recall that, for a complex $C$, the shifted complex $\Sigma^{n} C$ is defined to be the complex with $\left(\Sigma^{n} C\right)_{i}=C_{i-n}$ and $d_{i}^{\Sigma^{n} C}=(-1)^{n} d_{i-n}^{C}$. For objects $X, Y \in D^{b}(A)$, the cohomology groups $\operatorname{Ext}_{A}^{i}(X, Y)$ are 
defined by $\operatorname{Hom}_{D^{b}(A)}\left(X, \Sigma^{i} Y\right)$. For $A$-modules $X$ and $Y$ this definition reduces to the usual Ext. We write $\operatorname{Ext}_{A}(X, Y)$ for $\oplus_{i \geq 0} \operatorname{Ext}_{A}^{i}(X, Y)$. Then $\operatorname{Ext}_{A}(X, X)$ is a graded ring and $\operatorname{Ext}_{A}(X, Y)$ is a $\operatorname{Ext}_{A}(Y, Y)$ $\operatorname{Ext}_{A}(X, X)$-bimodule via composition products.

Central actions on cohomology. Let $H=\oplus_{n \geq 0} H_{n}$ be a positively graded ring which is graded-commutative, that is, $\eta \theta=(-1)^{|\eta||\theta|} \theta \eta$ for all homogeneous elements $\eta, \theta \in H$. We say that $H$ acts centrally on $D^{b}(A)($ cf., $[6,10,12])$ if, for every complex $X \in D^{b}(A)$, there is a graded (homologically) ring homomorphism

$$
H \stackrel{\varphi_{X}}{\longrightarrow} \operatorname{Ext}_{A}(X, X)
$$

and for every complex $Y$ and all homogeneous elements $\eta \in H, \theta \in$ $\operatorname{Ext}_{A}(X, Y)$, the equality $\varphi_{Y}(\eta) \circ \theta=(-1)^{|\eta||\theta|} \theta \circ \varphi_{X}(\eta)$ holds. In other words, the left and right $H$-module structures on $\operatorname{Ext}_{A}(X, Y)$ coincide up to a sign.

We need two important definitions pertaining to these rings of central cohomology actions. The first addresses commutativity of the central actions with dualization, and the second is our finiteness condition.

The duality anti-isomorphism. Let $C$ be a totally acyclic complex of free modules with $M=M_{C}$. Then distinct homology classes in $\operatorname{Ext}_{A}^{n}(M, M)$ correspond bijectively to distinct homotopy equivalence classes of chain maps $C \rightarrow \Sigma^{n} C$. Dualizing, we obtain a chain map $\Sigma^{-n}\left(C^{*}\right)=\left(\Sigma^{n} C\right)^{*} \rightarrow C^{*}$. Applying the shift functor $\Sigma^{n}$ to this chain map we now get a chain map $C^{*} \rightarrow \Sigma^{n}\left(C^{*}\right)$, and this corresponds to an element in $\operatorname{Ext}_{A}^{n}\left(M^{*}, M^{*}\right)$. One checks easily that this defines an anti-isomorphism

$$
\mathcal{D}: \operatorname{Ext}_{A}(M, M) \longrightarrow \operatorname{Ext}_{A}\left(M^{*}, M^{*}\right)
$$

of graded rings.

Definition 2.2. Let $M$ be an $A$-module of G-dimension zero. We say that a central ring action from $H$ on $D^{b}(A)$ commutes with dualization 
of $M$, provided that the diagram

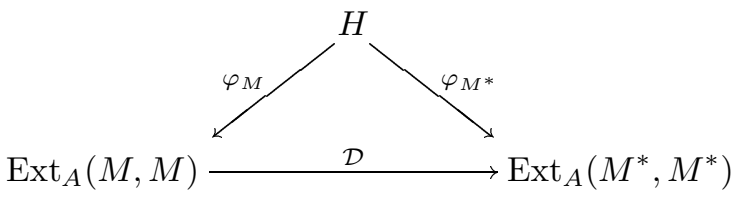

commutes, that is, $\mathcal{D}\left(\varphi_{M}(\eta)\right)=\varphi_{M^{*}}(\eta)$ for every homogeneous element $\eta \in H$.

The following fact describes in more detail how one may represent $\mathcal{D}(\eta)$ for $\eta \in \operatorname{Ext}_{A}(M, M)$.

Fact 2.3. Let $C$ and $D$ be totally acyclic complexes, and $M=M_{C}$, $N=M_{D}$. Then an element $\eta \in \operatorname{Ext}_{A}(M, N)$ can be represented for any $i \in \mathbf{Z}$ by a map $f_{\eta}: \Omega_{A}^{i+|\eta|}(M) \rightarrow \Omega_{A}^{i}(N)$. Conversely, for any $i \in \mathbf{Z}$ and $j \geq 0$, a map $f: \Omega_{A}^{i+j}(M) \rightarrow \Omega_{A}^{i}(N)$ represents an element of $\operatorname{Ext}_{A}^{j}(M, N)$. Two maps $f: \Omega_{A}^{i+j}(M) \rightarrow \Omega_{A}^{i}(N)$ and $g: \Omega_{A}^{i^{\prime}+j}(M) \rightarrow \Omega_{A}^{i^{\prime}}(N)$ represent the same element of $\operatorname{Ext}_{A}^{j}(M, N)$ if the following holds. Without loss of generality, assume that $i \geq i^{\prime}$. Lifting $g$ we obtain a chain map $C_{\geq i^{\prime}+j} \rightarrow D_{\geq i^{\prime}}$, and therefore a map $g^{\prime}: \Omega_{A}^{i+j}(M) \rightarrow \Omega_{A}^{i}(N)$. Then $f$ and $g$ represent the same element of $\operatorname{Ext}_{A}^{j}(M, N)$ if $f-g^{\prime}$ is a cocycle, that is, if there exists a map $\sigma: C_{i+j-1} \rightarrow D_{i+j}$ such that $d_{i+j}^{D} \circ\left(f-g^{\prime}-\sigma \circ d_{i+j}^{C}\right)=0$.

Recalling that $\Omega_{A}^{i}(M)^{*}=\Omega_{A}^{-i}\left(M^{*}\right)$ for all $i \in \mathbf{Z}$, if

$$
f_{\eta}: \Omega_{A}^{i+|\eta|}(M) \longrightarrow \Omega_{A}^{i}(M)
$$

is a map representing the element $\eta \in \operatorname{Ext}_{A}(M, M)$, then the dual map

$$
\left(f_{\eta}\right)^{*}=\operatorname{Hom}_{A}\left(f_{\eta}, A\right): \Omega^{-i}\left(M^{*}\right) \longrightarrow \Omega_{A}^{-i-|\eta|}\left(M^{*}\right)
$$

represents the element $\mathcal{D}(\eta) \in \operatorname{Ext}_{A}\left(M^{*}, M^{*}\right)$.

Definition 2.4. We say that $\operatorname{Ext}_{A}(X, Y)$ is an eventually Noetherian $H$-module of finite length, and write $\operatorname{Ext}_{A}(X, Y) \in \operatorname{Noeth}^{\mathrm{fl}} H$, 
if the following holds: there is a number $n_{0}$ such that the $H$ module $\operatorname{Ext}_{A}^{\geq n_{0}}(X, Y)$ is Noetherian and the length $\ell_{H_{0}} \operatorname{Ext}_{A}^{n}(X, Y)$ of $\operatorname{Ext}_{A}^{n}(X, Y)$ as an $H_{0}$-module is finite for each $n \geq n_{0}$.

3. Symmetric growth. In this section we prove the main result on symmetric growth in minimal totally acyclic complexes of finitely generated free modules. In other words, we establish conditions such that the complexities of a G-dimension zero module and its dual are finite and equal. We start with the following well-known bit of homological algebra which we will need in the course of the proof of the main theorem.

Fact 3.1. Let $C$ and $D$ be totally acyclic complexes over $A, X=M_{C}$, $Y=M_{D}$, and let $Z$ be an $A$-module. Choose a homogeneous element $\eta \in \operatorname{Ext}_{A}(X, Y)$, and let $f_{\eta}: \Omega_{A}^{i+|\eta|}(X) \rightarrow \Omega_{A}^{i}(Y)$ be a homomorphism representing $\eta$ for some $i \in \mathbf{Z}$, according to Fact 2.3. Given a commutative diagram

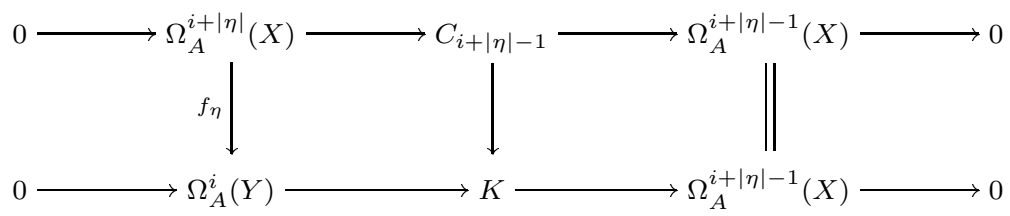

the induced long exact sequence of cohomology for $n \gg 0$ on the bottom row is

$$
\begin{aligned}
\cdots \longrightarrow \operatorname{Ext}_{A}^{n-i}(K, Z) \longrightarrow \operatorname{Ext}_{A}^{n}(Y, Z) & \stackrel{\partial_{n}}{\longrightarrow} \operatorname{Ext}_{A}^{n+|\eta|}(X, Z) \\
& \longrightarrow \operatorname{Ext}_{A}^{n-i+1}(K, Z) \longrightarrow \cdots
\end{aligned}
$$

where the connecting homomorphism $\partial_{n}$ is just right multiplication by $\eta$.

Similarly, given a commutative diagram

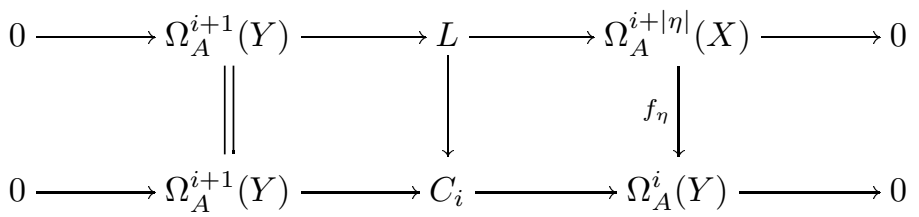


the induced long exact sequence of cohomology for $n \gg 0$ on the top row is

$$
\begin{aligned}
\cdots \longrightarrow \operatorname{Ext}_{A}^{n-i-1}(L, Z) \longrightarrow \operatorname{Ext}_{A}^{n}(Y, Z) & \stackrel{\partial_{n}}{\longrightarrow} \operatorname{Ext}_{A}^{n+|\eta|}(X, Z) \\
& \longrightarrow \operatorname{Ext}_{A}^{n-i}(L, Z) \longrightarrow \cdots
\end{aligned}
$$

where the connecting homomorphism $\partial_{n}$ is just pre-composition with $\eta$.

The next result is well-known; see [12, Remark 2.1], [1, Theorem 11.1] and [9, Proposition 5.3.2] for details.

Lemma 3.2. Let $(A, \mathfrak{m}, k)$ be a local ring, and let $M$ be a finitely generated $A$-module. Suppose that $\operatorname{Ext}_{A}(M, k)$ belongs to $\operatorname{Noeth}^{\mathrm{fl}} H$ for some graded-commutative ring $H$ acting centrally on $D^{b}(A)$. Then $\operatorname{cx}_{A} M$ is finite and the Poincaré series $P_{M}^{A}(t)=\sum_{n \geq 0} \beta_{n}(M) t^{n}$ is rational. Moreover, $\operatorname{cx}_{A} M$ equals the order of the pole of $P_{M}^{A}(t)$ at $t=1$.

In order to prove the main result, we also need the following elementary lemma. It shows that the category of modules of G-dimension zero, and the category of modules whose cohomology is finitely generated, are closed under extensions.

Lemma 3.3. Let $(A, \mathfrak{m}, k)$ be a local ring, and let

$$
0 \longrightarrow L \longrightarrow M \rightarrow N \rightarrow 0
$$

be an exact sequence of finitely generated A-modules.

(i) If $L$ and $N$ are both of $\mathrm{G}$-dimension zero, then so is $M$.

(ii) If $\operatorname{Ext}_{A}(L \oplus N, k)$ belongs to $\operatorname{Noeth}^{\mathrm{fl}} H$ for some graded-commutative ring $H$ acting centrally on $D^{b}(A)$, then so does $\operatorname{Ext}_{A}(M, k)$.

Proof. For a proof of (i), see for example [17, Lemma 1.1.10]. As for (ii), note that the exact sequence induces an exact sequence

$$
\operatorname{Ext}_{A}(N, k) \longrightarrow \operatorname{Ext}_{A}(M, k) \longrightarrow \operatorname{Ext}_{A}(L, k)
$$


of graded $H$-modules. It follows that the middle term must be eventually Noetherian of piecewise finite length.

Before stating the main theorem, recall that a full subcategory of $D^{b}(A)$ is thick if it is triangulated and closed under direct summands. The thick subcategory generated by a given object $X$, denoted thick $_{D^{b}(A)}(X)$, is the intersection of all the thick subcategories containing it.

Theorem 3.4. Let $(A, \mathfrak{m}, k)$ be a local ring, and $M$ a finitely generated $A$-module of $\mathrm{G}$-dimension zero. If $\operatorname{Ext}_{A}\left(M \oplus M^{*}, k\right)$ belongs to Noeth $^{\mathrm{fl}} H$ for some graded-commutative ring $H$ acting centrally on $D^{b}(A)$, such that its action commutes with dualization for all objects in thick $D^{b}(A)(M)$, then $\operatorname{cx}_{A} M=\operatorname{cx}_{A} M^{*}<\infty$.

Proof. We prove the result by induction on the complexity of $M$, which is finite by Lemma 3.2. If $\operatorname{cx}_{A} M=0$, then $M$ has finite projective dimension. Therefore by the Auslander-Bridger [2, (4.13)] and Auslander-Buchsbaum [14, 1.3.3] formulas we have that the Gdimension of $M$ and the projective dimension of $M$ are equal. Thus, $M$ is free. Therefore, so is $M^{*}$, and hence $\operatorname{cx}_{A} M^{*}=0$.

Next, suppose that $\operatorname{cx}_{A} M$ is nonzero. By [12, Lemma 2.5], there exists a homogeneous element $\eta \in H$, of positive degree, inducing injective maps

$$
\begin{aligned}
\operatorname{Ext}_{A}^{n}(M, k) & \stackrel{\cdot \varphi_{M}(\eta)}{\longrightarrow} \operatorname{Ext}_{A}^{n+|\eta|}(M, k) \\
\operatorname{Ext}_{A}^{n}\left(M^{*}, k\right) & \stackrel{\cdot \varphi_{M *}(\eta)}{\longrightarrow} \operatorname{Ext}_{A}^{n+|\eta|}\left(M^{*}, k\right)
\end{aligned}
$$

for $n \gg 0$. Choose a minimal totally acyclic complex $C$ such that $M=M_{C}$ and a map $f_{\varphi_{M}(\eta)}: \Omega_{A}^{|\eta|}(M) \rightarrow M$ representing the element $\varphi_{M}(\eta)$ in $\operatorname{Ext}_{A}^{|\eta|}(M, M)$. Consider the pushout diagram:

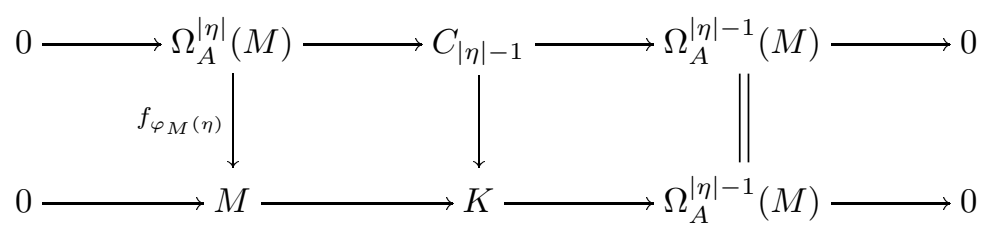


Dualizing this diagram, using the fact that $\operatorname{Ext}_{A}^{1}(M, A)=\operatorname{Ext}_{A}^{1}\left(\Omega_{A}(M)\right.$, $A)=0,\left(C^{*}\right)_{n}=\left(C_{-n-1}\right)^{*}$ and $\Omega_{A}^{i}(M)^{*}=\Omega_{A}^{-i}\left(M^{*}\right)$, we obtain the commutative diagram with exact rows:

$(\ddagger)$

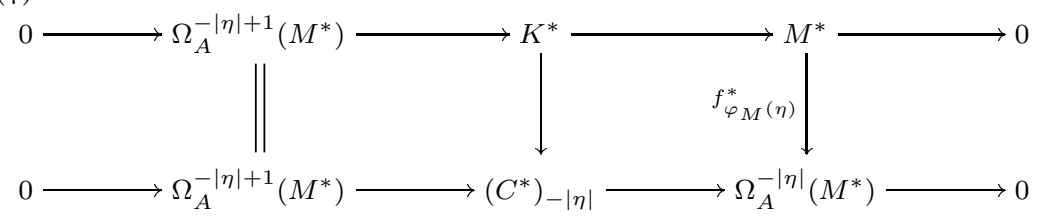

Since both the end term modules in the bottom row of $(\dagger)$ have Gdimension zero, so does the module $K$ by [17, Lemma 1.1.10], and therefore so does $K^{*}$. Moreover, since the $H$-module $\operatorname{Ext}_{A}(X, k)$ belongs to Noeth ${ }^{\mathrm{fl}} H$ whenever $X$ is one of the end term modules in

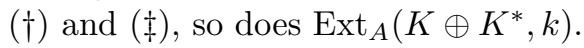

The bottom row of $(\dagger)$ induces a long exact sequence for all $n \gg 0$

$$
\begin{aligned}
\cdots \longrightarrow \operatorname{Ext}_{A}^{n}(K, k) \longrightarrow \operatorname{Ext}_{A}^{n}(M, k) \stackrel{\partial_{n}}{\longrightarrow} & \operatorname{Ext}_{A}^{n+|\eta|}(M, k) \\
& \longrightarrow \operatorname{Ext}_{A}^{n+1}(K, k) \longrightarrow \cdots
\end{aligned}
$$

in cohomology. By Fact 3.1, the connecting homomorphism $\partial_{n}$ is just scalar multiplication with $\varphi_{M}(\eta)$, and is therefore injective for all $n \gg 0$. The top row of $(\ddagger)$ also induces a cohomological long exact sequence for all $n \gg 0$, which takes the form

$$
\begin{aligned}
\cdots \longrightarrow \operatorname{Ext}_{A}^{n-1}\left(K^{*}, k\right) \longrightarrow \operatorname{Ext}_{A}^{n-|\eta|}\left(M^{*}, k\right) & \stackrel{\partial_{n}^{\prime}}{\longrightarrow} \operatorname{Ext}_{A}^{n}\left(M^{*}, k\right) \\
& \longrightarrow \operatorname{Ext}_{A}^{n}\left(K^{*}, k\right) \longrightarrow \cdots
\end{aligned}
$$

Moreover, by Facts 2.3 and 3.1, for such $n$, the connecting homomorphism $\partial_{n}^{\prime}$ is scalar multiplication with $\mathcal{D}\left(\varphi_{M}(\eta)\right)$. By assumption, the central ring action from $H$ on $D^{b}(A)$ commutes with dualization of $M$, that is, $\mathcal{D}\left(\varphi_{M}(\eta)\right)=\varphi_{M *}(\eta)$. Therefore the connecting homomorphism $\partial_{n}^{\prime}$ is also injective for all $n \gg 0$.

Choose a number $n_{0} \geq|\eta|$ with the property that both $\partial_{n}$ and $\partial_{n}^{\prime}$ are injective for $n \geq n_{0}$. Then the sequences

$$
\begin{aligned}
& 0 \longrightarrow \operatorname{Ext}_{A}^{n}(M, k) \longrightarrow \operatorname{Ext}_{A}^{n+|\eta|}(M, k) \longrightarrow \operatorname{Ext}_{A}^{n+1}(K, k) \longrightarrow 0 \\
& 0 \longrightarrow \operatorname{Ext}_{A}^{n-|\eta|}\left(M^{*}, k\right) \longrightarrow \operatorname{Ext}_{A}^{n}\left(M^{*}, k\right) \longrightarrow \operatorname{Ext}_{A}^{n}\left(K^{*}, k\right) \longrightarrow 0
\end{aligned}
$$


are exact for $n \geq n_{0}$, giving equalities

$$
\begin{aligned}
\beta_{n+1}(K) & =\beta_{n+|\eta|}(M)-\beta_{n}(M) \\
\beta_{n}\left(K^{*}\right) & =\beta_{n}\left(M^{*}\right)-\beta_{n-|\eta|}\left(M^{*}\right)
\end{aligned}
$$

of Betti numbers. Computing Poincaré series, we obtain

$$
\begin{aligned}
P_{K}^{A}(t) & =\frac{\left(1-t^{|\eta|}\right) P_{A}(M, t)}{t^{|\eta|-1}}+f(t) \\
P_{K^{*}}^{A}(t) & =\left(t^{|\eta|}-1\right) P_{A}\left(M^{*}, t\right)+g(t)
\end{aligned}
$$

for some polynomials $f(t), g(t) \in \mathbf{Z}[t]$. Consequently, the order of the pole of $P_{K}^{A}(t)$ at $t=1$ is one less than that of $P_{M}^{A}(t)$, whereas the pole of $P_{K^{*}}^{A}(t)$ is one less than that of $P_{M^{*}}^{A}(t)$. Therefore, by Lemma 3.2, the complexity of $K$ is $\operatorname{cx}_{A} M-1$, whereas the complexity of $K^{*}$ is $\operatorname{cx}_{A} M^{*}-1$. As an object of $D^{b}(A)$, the module $K$ belongs to thick $_{D^{b}(A)}(M)$, and so by induction we obtain

$$
\operatorname{cx}_{A} M=\operatorname{cx}_{A} K+1=\operatorname{cx}_{A} K^{*}+1=\operatorname{cx}_{A} M^{*} .
$$

This completes the proof.

4. The ring of cohomology operators. When $M$ is an $A$-module of finite complete intersection dimension (see the definition below) then one has a ring $H$ acting centrally on $D^{b}(A)$, namely the polynomial ring of cohomology operators

$$
H=A\left[\chi_{1}, \ldots, \chi_{c}\right]
$$

with each cohomology operator $\chi_{i}$ of degree two. Inferred from the main theorem of Gulliksen $[\mathbf{1 9}]$ is the fact that $\operatorname{Ext}_{A}(M, M)$ is in Noeth $^{\mathrm{fl}} H$. In this section we show that, for modules of complete intersection dimension zero, the action of the ring of cohomology operators commutes with dualization. It then follows from Theorem 3.4 that $\operatorname{cx}_{A} M=\operatorname{cx}_{A} M^{*}<\infty$ for such a module $M$. A consequence of this is that if $C$ is a totally acyclic complex, and any image in $C$ has finite complete intersection dimension, then $C$ has symmetric polynomial growth.

For the purposes below, we recall the definition of the elements $\varphi_{M}\left(\chi_{i}\right) \in \operatorname{Ext}_{A}^{2}(M, M)$, and their action on $\operatorname{Ext}_{A}(M, N)$ and $\operatorname{Ext}_{A}(N$, 
$M)$. (There are actually several definitions for these elements, but they all agree up to sign; see [8]. The one we give is from [18].)

Assume that $A=B /\left(x_{1}, \ldots, x_{c}\right)$, where $(B, \mathfrak{n})$ is a local ring and $x_{1}, \ldots, x_{c}$ is a $B$-regular sequence contained in $\mathfrak{n}$. Let $(C, d)$ be a complex of finitely generated free $A$-modules. Choose free $B$-modules $\widetilde{C}_{i}$ and maps $\widetilde{d}_{i}$ between them such that $\left(\widetilde{C} \otimes_{B} A, \widetilde{d} \otimes_{B} A\right)$ and $(C, d)$ are isomorphic complexes. Since $\widetilde{d}^{2} \equiv 0$ modulo $\left(x_{1}, \ldots, x_{c}\right)$, we can decompose $\widetilde{d}^{2}$ as

$$
\widetilde{d}^{2}=\sum_{i=1}^{c} x_{i} \widetilde{t}_{i}
$$

for some family $\left(\widetilde{t}_{i}\right)_{i=1}^{c}$ of degree -2 endomorphisms of the graded $B$-module $\widetilde{C}$. Then $t_{i}=\widetilde{t}_{i} \otimes_{B} A$ become degree -2 chain maps on the complex $C$ which are well defined and commute up to homotopy (see [18]). The chain maps $t_{i}$ on a free resolution $C$ of $M$ then define the elements $\varphi_{M}\left(\chi_{i}\right) \in \operatorname{Ext}_{A}^{2}(M, M)$. The action of $\varphi_{M}\left(\chi_{i}\right)$ on $\operatorname{Ext}_{A}(M, N)$ and $\operatorname{Ext}_{A}(N, M)$ is thus determined by composition of chain maps. If $M=M_{C}$ for a totally acyclic complex $C$ of free $A$-modules, then the element $\varphi_{M}\left(\chi_{i}\right) \in \operatorname{Ext}_{A}^{2}(M, M)$ is determined by the chain map $t_{i}: C \rightarrow \Sigma^{2} C$.

Recall that a quasi-deformation of local rings is a diagram

$$
A \rightarrow A^{\prime} \leftarrow B
$$

of local homomorphisms satisfying the following: the map $A \rightarrow A^{\prime}$ is flat, and $A^{\prime} \leftarrow B$ is surjective with kernel generated by a $B$-regular sequence contained in the maximal ideal of $B$. If $M$ is a finitely generated module over $A$, then its complete intersection dimension, denoted CI- $\operatorname{dim}_{A} M$, is defined as

$$
\begin{aligned}
\text { CI- } \operatorname{dim}_{A} M \stackrel{\text { def }}{=} \inf \left\{\operatorname{pd}_{B}\left(M \otimes_{A} A^{\prime}\right)-\operatorname{pd}_{B} A^{\prime} \mid\right. \\
\left.A \rightarrow A^{\prime} \leftarrow B \text { is a quasi-deformation }\right\} .
\end{aligned}
$$

This was introduced in [5], where it was shown that this invariant dominates the G-dimension and satisfies the "Auslander-Bridger formula." Namely, if $M$ has finite CI-dimension, then it also has finite G-dimension and

$$
\text { CI-dim }{ }_{A} M=\mathrm{G}-\operatorname{dim}_{A} M=\operatorname{depth} A-\operatorname{depth} M .
$$


We can now state the main theorem of this section.

Theorem 4.1. Let $A=B /\left(x_{1}, \ldots, x_{c}\right)$, where $(B, \mathfrak{n}, k)$ is a local ring and $x_{1}, \ldots, x_{c}$ is a $B$-regular sequence contained in $\mathfrak{n}$. Suppose that $M$ is an $A$-module with $\operatorname{depth}_{A} M=\operatorname{depth}_{A} A$, and $\operatorname{pd}_{B} M<\infty$. Then the central ring action of the polynomial ring of cohomology operators $H=A\left[\chi_{1}, \ldots, \chi_{c}\right]$ on $D^{b}(A)$ commutes with dualization of $M$.

Proof. The hypotheses show that the CI-dimension of $M$ is zero, and therefore $M$ has G-dimension zero. Let $C$ be a totally acyclic complex of free modules with $M=M_{C}$. Choose $\chi=\chi_{i} \in H$. It suffices to prove that $\mathcal{D}\left(\varphi_{M}(\chi)\right)=\varphi_{M^{*}}(\chi)$. The element $\varphi_{M}(\chi) \in \operatorname{Ext}_{A}(M, M)$ is determined by the chain map $t: C \rightarrow \Sigma^{2} C$, as described above. Therefore $\mathcal{D}\left(\varphi_{M}(\chi)\right)$ corresponds to the chain map $\sigma^{2} t^{*}: C^{*} \rightarrow \Sigma^{2} C^{*}$. On the other hand, if one uses the lifting $\operatorname{Hom}_{B}(\widetilde{C}, B)$ of $C^{*}$ with lifted maps $(\widetilde{d})^{*}=\operatorname{Hom}_{A}(\widetilde{d}, B)$, and the factorization of $\left((\widetilde{d})^{*}\right)^{2}$ dual to that of $\widetilde{d}^{2}$, we see that the chain map $\Sigma^{2} t^{*}: C^{*} \rightarrow \Sigma^{2} C^{*}$ defines the element $\varphi_{M^{*}}(\chi) \in \operatorname{Ext}_{A}\left(M^{*}, M^{*}\right)$, and this is what we wanted to show.

Remark. As with the notion of G-dimension, the notion of complete intersection dimension makes sense for complexes in the bounded derived category (cf. [21]). Not surprisingly, Theorem 4.1 holds for complexes of finite CI-dimension. Moreover, given such a complex $X$ in $D^{b}(A)$, every complex in thick $D^{b}(A)(X)$ also has finite CI-dimension, over the same quasi-deformation as $X$ (cf. [11]).

As a consequence of Theorem 4.1 and the above remark, we obtain the following result, which is an application of Theorem 3.4 in the case when the module has CI-dimension zero. (cf. [5, 3.3] which can be used to give an alternate proof using the notion of support varieties).

Corollary 4.2. Let $(A, \mathfrak{m}, k)$ be a local ring, and $M$ a finitely generated $A$-module with CI- $\operatorname{dim}_{A} M=0$. Then $\operatorname{cx}_{A} M=\operatorname{cx}_{A} M^{*}$.

Proof. By [13, Lemma 3.5] and its proof, the CI-dimension of $M^{*}$ is also zero, and there exists a quasi deformation

$$
A \longrightarrow A^{\prime} \longleftarrow B
$$


such that $\operatorname{pd}_{B}\left(\left(M \oplus M^{*}\right) \otimes_{A} A^{\prime}\right)<\infty$. Moreover, from the isomorphism

$$
\operatorname{Hom}_{A}(M, A) \otimes_{A} A^{\prime} \cong \operatorname{Hom}_{A^{\prime}}\left(M \otimes_{A} A^{\prime}, A^{\prime}\right),
$$

we see that the complexity of the $A$-dual $M^{*}$ of $M$ equals that of the $A^{\prime}$ module $\operatorname{Hom}_{A^{\prime}}\left(M \otimes_{A} A^{\prime}, A^{\prime}\right)$, which is the $A^{\prime}$-dual of $M \otimes_{A} A^{\prime}$. Finally, the projective dimension of the $B$-module $\left(M \otimes_{A} A^{\prime}\right) \oplus \operatorname{Hom}_{A^{\prime}}\left(M \otimes_{A}\right.$ $\left.A^{\prime}, A^{\prime}\right)$ is finite, and the equality

$$
\operatorname{depth}_{A} A-\operatorname{depth}_{A} M=\operatorname{depth}_{A^{\prime}} A^{\prime}-\operatorname{depth}_{A^{\prime}}\left(M \otimes_{A} A^{\prime}\right)
$$

holds. Changing notation, we can therefore assume that $A=A^{\prime}$, and so $A=B /\left(x_{1}, \ldots, x_{c}\right)$, where $B$ is a local ring and $x_{1} \ldots, x_{c}$ is a $B$-regular sequence contained in the maximal ideal of $B$. Moreover, the projective dimension of the $B$-module $M \oplus M^{*}$ is finite, the G-dimension of $M$ is zero, and $\operatorname{depth}_{A} M=\operatorname{depth}_{A} A$. By Theorem 4.1 and the subsequent remark, the central ring action of the polynomial ring of cohomology operators $H=A\left[\chi_{1}, \ldots, \chi_{c}\right]$ on $D^{b}(A)$ commutes with dualization for every object in thick $D^{b}(A)(M)$. Therefore, by Theorem 3.4, it suffices to know that $\operatorname{Ext}_{A}\left(M \oplus M^{*}, k\right)$ belongs to $\operatorname{Noeth}^{\mathrm{fl}} H$, but this is implied by the main result of $[\mathbf{1 9}]$ (see also $[\mathbf{3}, \mathbf{1 8}]$ ).

Corollary 4.3. Let $(A, \mathfrak{m}, k)$ be a local ring and $C$ a totally acyclic minimal complex of finitely generated free $A$-modules. If $M_{C}$ has finite CI-dimension, then $C$ has symmetric growth.

Proof. Since $M_{C}$ is an infinite syzygy, its CI-dimension is zero by [5, Lemma 1.9].

When $A$ is a complete intersection, then every finitely generated $A$ module has finite CI-dimension. Therefore, in this case, every totally acyclic minimal complex of free modules has symmetric growth. This follows already from [3, Theorem 5.6], but, because of our alternative proof, we include it here as a corollary.

Corollary 4.4. Let $(A, \mathfrak{m}, k)$ be a local complete intersection. Then every totally acyclic minimal complex of finitely generated free $A$ modules has symmetric growth. 
We end this paper with a question on central ring actions and dualizations.

Question. Do there exist graded commutative rings $H$ acting centrally on $D^{b}(A)$ such that the action fails to commute with dualization of a finitely generated $A$-module $M$ ?

Acknowledgments. This work was done while the second author was visiting Trondheim, Norway, December-January, 2008-9. The second author thanks the Algebra Group at the Institutt for Matematiske Fag, NTNU, for their hospitality and generous support.

\section{REFERENCES}

1. M.F. Atiyah and I.G. Macdonald, Introduction to commutative algebra, Addison-Wesley, Boston, 1969.

2. M. Auslander and M. Bridger, Stable module theory, Mem. Amer. Math. Soc. 94, American Mathematical Society, Providence, R.I. 1969.

3. L. Avramov, Modules of finite virtual projective dimension, Invent. Math. 96 (1989), 71-101.

4. L. Avramov and R.-O. Buchweitz, Support varieties and cohomology over complete intersection, Invent. Math. 142 (2000), 285-318.

5. L. Avramov, V. Gasharov and I. Peeva, Complete intersection dimension, Inst. Haut. Etud. Sci. Publ. Math. 86 (1997), 67-114.

6. L. Avramov and S. Iyengar, Modules with prescribed cohomological support, Illinois J. Math. 51 (2007), 1-20.

7. L. Avramov and A. Martsinkovsky, Absolute, relative, and Tate cohomology of modules of finite Gorenstein dimension, Proc. Lond. Math. Soc. 85 (2002), 393-440.

8. L. Avramov and L-C. Sun, Cohomology operators defined by a deformation, J. Alg. 204 (1998), 684-710.

9. D.J. Benson, Representations and cohomology, Vol. II, Cambr. Stud. Adv. Math. 31, Cambridge University Press, Cambridge, 1991.

10. D.J. Benson, S. Iyengar and H. Krause, Local cohomology and support for triangulated categories, Ann. Sci. Ecol. Norm. 41 (2008), 573-619.

11. P.A. Bergh, On complexes of finite complete intersection dimension, Homology, Homotopy Appl. 11 (2009), 49-54.

12. P.A. Bergh, S. Iyengar, H. Krause and S. Oppermann, Dimensions of triangulated categories via Koszul objects, Math. Z. 265 (2010), 849-864.

13. P.A. Bergh and D. Jorgensen, On the vanishing of homology for modules of finite complete intersection dimension, J. Pure Appl. Alg. 215 (2011), 242-252. 
14. W. Bruns and J. Herzog, Cohen-Macaulay rings, Cambr. Stud. Adv. Math. 39, Cambridge University Press, Cambridge, 1993.

15. R.-O. Buchweitz, Maximal Cohen-Macaulay modules and Tate cohomology over Gorenstein rings, University of Hannover, 1986.

16. R.-O. Buchweitz and H. Flenner, Global Hochschild (co-)homology of singular spaces, Adv. Math. 217 (2008), 205-242.

17. L.W. Christensen, Gorenstein dimensions, Lect. Notes Math. 1747, SpringerVerlag, New York, 2000.

18. D. Eisenbud, Homological algebra on a complete intersection with an application to group representations, Trans. Amer. Math. Soc. 260 (1980), 35-64.

19. T.H. Gulliksen, A change of ring theorem with applications to Poincaré series and intersection multiplicity, Math. Scand. 34 (1974), 167-183.

20. D.A. Jorgensen and L.M. Şega, Asymmetric complete resolutions and vanishing of Ext over Gorenstein rings, Int. Math. Res. Not. 2005 (2005), 3459-3477.

21. S. Sather-Wagstaff, Complete intersection dimensions for complexes, J. Pure Appl. Alg. 190 (2004), 267-290.

Institutt for Matematiske Fag, NTNU, N-7491 Trondheim, Norway

Email address: bergh@math.ntnu.no

Department of Mathematics, University of Texas at Arlington, ArlingTON, TX 76019

Email address: djorgens@uta.edu 